\title{
BILATERAL AGGRESSIVE ANGIOMYXOMA IN PREGNANCY
}

Seetesh Ghose, Dilip K. Maurya, R. Narasimhan, Anitha Manoj

1. Professor, Department of Obstetrics and Gynaecology, Mahatma Gandhi Medical College and Research Institute, Pilliyarkuppam, Pondicherry.

2. Assistant Professor, Department of Obstetrics and Gynaecology, Mahatma Gandhi Medical College and Research Institute, Pilliyarkuppam, Pondicherry.

3. Professor, Department of Pathology, Mahatma Gandhi Medical College and Research Institute, Pilliyarkuppam, Pondicherry.

4. Assistant Professor, Department of Obstetrics and Gynaecology, Mahatma Gandhi Medical College and Research Institute, Pilliyarkuppam, Pondicherry.

\section{CORRESPONDING AUTHOR}

Seetesh Ghose,

Professor \& Head,

Dept. of O\&G

Mahatma Gandhi Medical College and Research Institute,

Pilliyarkuppam, Pondicherry

E-mail: seetesh@mgmcri.ac.in

Ph: 00919443244630

ABSTRACT: A 22 Year, primigravida presented with swelling and pain at the labia minora for one month. As she did not respond to the conservative management, that swelling was excised . The histopathology report came as aggressive angiomyxoma. Patient had an uncomplicated vaginal delivery.

KEY WORDS: vulval angiomyxoma, pregnancy, bilateral

INTRODUCTION: Aggressive angiomyxoma is a predominantly non-metastasizing, locally infiltrative soft tissue neoplasm. Most commonly it is encountered in the vulvovaginal region of women of reproductive age, during the third to fifth decades. Patients often present with vague symptoms secondary to pressure effects on the adjacent urogenital or anorectal tracts. The clinical impression frequently includes Bartholin gland cyst or hernia of canal of Knuck and often underestimates the extent of disease. The treatment of choice is wide local excision with attention to margins. In view of its rare association with pregnancy and bilateralism, we present this case report to the committee of scientific forum.

CASE REPORT: A 22 yr old primigravida presented with approximately 34 weeks of amenorrhea and complaints of swelling in the external genitalia since 1 month. The swelling was insidious in onset, progressive in nature and associated with pain. Pain is pricking type, aggravates on walking and doesn't decrease on resting. She also says that there was no pain associated with the swelling to start with, pain developed only after she sought medical help locally in her place, where they did multiple punctures over the swelling with needle. No history of fever, bowel or bladder disturbance, trauma, weight loss. She was not sure of her last menstrual period and had regular normal previous menstrual cycles. Trimesters of present pregnancy were uneventful.

On examination, she was pale with bilateral pitting pedal edema and no palpable lymph nodes. She had stable vital signs. Per abdomen examination showed 34 weeks sized relaxed uterus with single fetus in cephalic presentation, adequate liquor and good fetal heart sounds of 
150-160 beats per minute. External genitalia showed 2 pedunculated swellings around 4X3X2 $\mathrm{cm}$ and $5 \mathrm{X} 4 \mathrm{X} 3 \mathrm{~cm}$ arising from each labia minora with smooth surface and rounded borders. Skin over the swelling was tense, glossy and edematous (Fig.1). No visible cough impulse and no visible peristalsis. Swelling was tender with no local rise of temperature and was uniformly soft in consistency, not reducible, not fixed to the overlying skin. Fluctuation and transillumination were absent. Per speculam \& per vaginal examination showed uneffaced cervix with closed external os. Provisional diagnosis of Primigravida at 32-34 weeks of gestation with pedunculated vulvaledema was made. Investigations revealed hemoglobin of $7.5 \mathrm{gm} \%$ and all other parameters were normal. Ultrasound of the abdomen showed a single live intrauterine gestation corresponding to 34 weeks. The swelling was treated with glycerine magnesium sulphate local dressings for which patient showed no improvement. In view of patient's discomfort and to provide her relief at the earliest the swelling was excised and sent for histopathological examination. Histopathological report showed Aggressive angiomyxoma. After 4-5 weeks of excision, patient had spontaneous vaginal delivery at term with minimal interventions. Patient has been counselled about the recurrence and metastasis, therefore the need for follow up at least once a year for five years.

DISCUSSION: Aggressive angiomyxoma was first described by Steeber and Rosai in 1983.1Using the MEDLINE search engine, just over 100 cases of aggressive angiomyxoma have been reported in women in the world literature. The term "aggressive" has been applied to this tumor secondary to its propensity for recurrence. It is a rare mesenchymal tumor most often found in the pelvis, perineum, vulva, buttock, retroperitoneum, and inguinal regions. Cases of aggressive angiomyxoma (AAM) have also been described in males with a female to male ratio of 6.6:1.2 Diagnosis is usually made in the fourth decade of life with ages ranging from 16 to 70 years at the time of the initial surgical procedure. ${ }^{3}$ The size of these tumors is extremely variable, ranging from $1 \mathrm{~cm}$ up to $60 \mathrm{~cm} .^{2}$ The diagnosis of AAM is a difficult one to make with preoperative misdiagnosis occurring in $>82 \%$ of cases. ${ }^{4}$

Symptoms are often vague and include pain in the perineal, labial, or pelvic region, pressure-like pulsating sensations, dyspareunia, and sensation of a pelvic mass with heavy lifting or menstruation and general pelvic and abdominal fullness. ${ }^{3}$ Physical examination will reveal a vulvar, perineal, or pelvic mass in most cases. However, physical examination will almost always underestimate the full extent of the AAM except in cases of small vulvar or vaginal lesions. ${ }^{4}$ Alternative diagnoses include vulvar abscess, Bartholin's cyst, Gartner's duct cyst, vaginal prolapse, pelvic floor hernia, vaginal mass or polyp, obturator or levator hernia, liposarcoma, fibrosarcoma, and sarcoma botryoides. .5

On gross examination, these tumors have been described as poorly encapsulated masses with an adherence to fat, fibrous tissue, and muscle. The tumors have anywhere from a soft to solid consistency with a pink or reddish tan external surface. The cut surface of these tumors has been described as gelatinous, edematous, and cystic with a mucoid or watery content. Necrotic and hemorrhagic tissue has been described on cross-section examination of the tumor. Histopathologically, AAMs are derived from myofibroblasts. The neoplastic cells have been reported to be benign-appearing spindle or stellate cells in a myxoid stroma surrounded by numerous capillaries ${ }^{7,8}$. The cells themselves are generally hyperchromatic with dense homogenous chromatin, occasional nucleoli, and few if any mitoses. Large- and medium-sized blood vessels have been described scattered throughout the stoma with small capillary-sized vessels also present. The largest study showed immunoreactivity for vimentin and desmin in 
$100 \%$ of tumors tested. Most of these tumors contained tumor cells with focal reactivity for muscle-specific actin and smooth muscle actin. Less than $25 \%$ of tumors cells stained positive for CD-34. Staining for S-100 was negative in all cases. ${ }^{3}$ The estrogen and progesterone receptor status of these tumors has been reported in multiple studies.

The principle treatment of AAM in most cases has been surgery with the primary goal being to obtain clear margins of resection. Surgery is not feasible and mutilating when it is large and retroperitoneal. Multiple case reports suggest that medical management with a GnRH agonist is a viable option for both primary and recurrent tumors. Chemotherapy and radiation are ineffective as an adjuvant treatment to surgery.

In almost all cases reported in the literature, AMM is described as a non-metastatic tumor that does not result in the death of the patient. There are 2 relatively recent reports that describe metastatic aggressive angiomyxomas with a resultant death in one of the patients. ${ }^{9}$, ${ }^{10}$ Both reports describe a primary pelvic or perineal AAM with a biopsy-proven metastatic tumor at a distant sight (lung and mediastinum). The secondary tumors were diagnosed by histology and/or immunohistochemistry. The uniqueness of this case is its bilaterallity and occurrence during the pregnancy which has not yet reported.

\section{REFERENCES:}

1. Steeper TA, Rosia J. Aggressive angiomyxoma of the female pelvis and perineum: report of nine cases of a distinctive type of gynecologic soft tissue neoplasm. Am J ClinPathol. 1983; 7:453.

2. Chan IM, Hon E, Ngai SW, et al. Aggressive angiomyxoma in females: is radical resection the only option? ActaObstetGynecol Scand. 2000; 79:216-220.

3. Fetsch JF, Laskin WB, Lefkowitz M, et al. Aggressive angiomyxoma, a clinicopathologic study of 29 female patients. Cancer. 1996; 78:79-90.

4. Smith $\mathrm{OH}$, Worrel RV, Smith AY, et al. Aggressive angiomyxoma of the female pelvis and peritoneum review of the literature. GynecolOncol. 1991; 42:79-85.

5. Gungor T, Zengeroglu S, Kaleli A, et al. Aggressive angiomyxoma of the vulva and vagina a common problem: misdiagnosis. Eur J ObstetGynecolReprod Biol. 2004; 112:114-116.

6. Melilli GA, Carriero C, Nappi L, et al. Aggressive angiomyxoma of the pelvis: a case report. Minerva Ginecol. 2000; 53:465-456.

7. Martinez MA, Ballestin C, Carabias E, et al. Aggressive angiomyxoma: an ultrastructure study of four cases. UltrastructPathol. 2003; 27:227-233.

8. Yalinkaya A, Askar I, Bayhan G, et al. Aggressive angiomyxoma of the female pelvis and the labium. ActaObstetGynecol Scand. 2003; 82:298-301.

9. Siassi RM, Papadopoulos T, Matzel KE. Metastasizing aggressive angiomyxoma. N Engl J Med. 1999; 2:1772.

10. Blandamura S, Cruz J, Faure Veraga L, et al. Aggressive angiomyxoma: a second case of metastasis with a patient's death. Hum Pathol. 2003; 34:1072-1074. 